\title{
Results on Planar Parallel Manipulators with Cylindrical Singularity Surface
}

\author{
G. Nawratil \\ Institute of Discrete Mathematics and Geometry, Vienna University of Technology, Austria, \\ e-mail: nawratil@geometrie.tuwien.ac.at
}

\begin{abstract}
In this article we give first results on Stewart Gough Platforms with planar base and platform, whose singularity set for any orientation of the platform is a cylindrical surface with rulings parallel to a given fixed direction $p$ in the space of translations. In this case the singularity set can easily be visualized as curve by choosing $p$ as projection direction. Moreover the computation of singularity free zones reduces to a 5-dimensional task. We prove that there do not exist non-architecturally singular Stewart Gough Platforms with planar base and platform and no four anchor points collinear which possess such a singularity surface.
\end{abstract}

Key words: Stewart Gough Platform, planar parallel manipulator, cylindrical singularity surface, architecture singular manipulators

\section{Introduction}

The geometry of the parallel manipulator is given by the six base anchor points $\mathbf{M}_{i}:=$ $\left(A_{i}, B_{i}, C_{i}\right)^{T}$ in the fixed space and by the six platform anchor points $\mathbf{m}_{i}:=\left(a_{i}, b_{i}, c_{i}\right)^{T}$ in the moving space. By using Euler Parameters $\left(e_{0}, e_{1}, e_{2}, e_{3}\right)$ for the parametrization of the spherical motion group the coordinates $\mathbf{m}_{i}^{\prime}$ of the platform anchor points with respect to the fixed space can be written as $\mathbf{m}_{i}^{\prime}=K^{-1} \mathbf{R} \cdot \mathbf{m}_{i}+\mathbf{t}$ with

$$
\mathbf{R}:=\left(r_{i j}\right)=\left(\begin{array}{ccc}
e_{0}^{2}+e_{1}^{2}-e_{2}^{2}-e_{3}^{2} & 2\left(e_{1} e_{2}+e_{0} e_{3}\right) & 2\left(e_{1} e_{3}-e_{0} e_{2}\right) \\
2\left(e_{1} e_{2}-e_{0} e_{3}\right) & e_{0}^{2}-e_{1}^{2}+e_{2}^{2}-e_{3}^{2} & 2\left(e_{2} e_{3}+e_{0} e_{1}\right) \\
2\left(e_{1} e_{3}+e_{0} e_{2}\right) & 2\left(e_{2} e_{3}-e_{0} e_{1}\right) & e_{0}^{2}-e_{1}^{2}-e_{2}^{2}+e_{3}^{2}
\end{array}\right)
$$

the translation vector $\mathbf{t}:=\left(t_{1}, t_{2}, t_{3}\right)^{T}$ and $K:=e_{0}^{2}+e_{1}^{2}+e_{2}^{2}+e_{3}^{2}$. Moreover it should be noted that $K$ is used as homogenizing factor whenever it is suitable.

It is well known (see e.g. [5]) that the set of singular configurations is given by $Q:=$ $\operatorname{det}(\mathbf{Q})=0$, where the $i^{t h}$ row of the $6 \times 6$ matrix $\mathbf{Q}$ equals the Plïcker coordinates $\left(\mathbf{l}_{i}, \widehat{\mathbf{l}_{i}}\right):=$ $\left(\mathbf{R} \cdot \mathbf{m}_{i}+\mathbf{t}-K \mathbf{M}_{i}, \mathbf{M}_{i} \times \mathbf{l}_{i}\right)$ of the carrier line of the $i^{\text {th }}$ leg.

As we consider only manipulators with planar platform we may suppose $c_{i}=0$ for $i=1, \ldots, 6$. We set up the planar base in a more general position as

$$
C_{1}=0, \quad C_{i}=\left[C_{2}\left(B_{3} A_{i}-A_{3} B_{i}\right)+A_{2} C_{3} B_{i}\right] /\left(A_{2} B_{3}\right) \quad \text { for } \quad i=4,5,6 \text {. }
$$

Moreover it was proven by Karger in [2] that for planar parallel manipulator with no four points on a line we can assume $A_{1}=B_{1}=B_{2}=a_{1}=b_{1}=b_{2}=0$ and $A_{2} B_{3} B_{4} B_{5} a_{2}\left(a_{4}-\right.$ $\left.a_{3}\right) \operatorname{coll}(3,4,5) \neq 0$ with

$$
\operatorname{coll}(i, j, k):=a_{i}\left(b_{j}-b_{k}\right)+a_{j}\left(b_{k}-b_{i}\right)+a_{k}\left(b_{i}-b_{j}\right) .
$$

Note that $\operatorname{coll}(i, j, k)=0$ characterizes collinear platform anchor points $\mathbf{m}_{i}, \mathbf{m}_{j}$ and $\mathbf{m}_{k}$. 


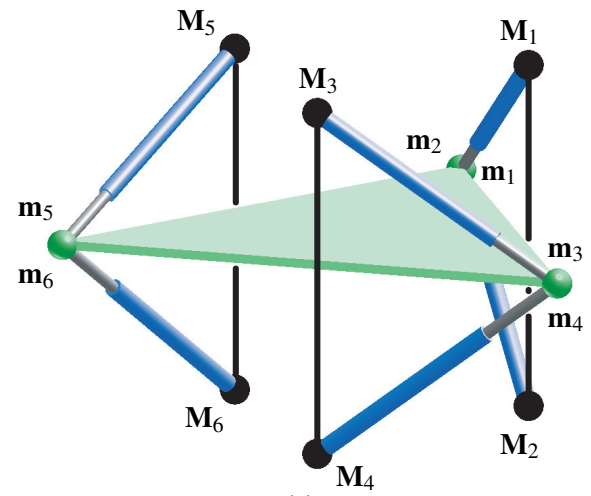

(a)

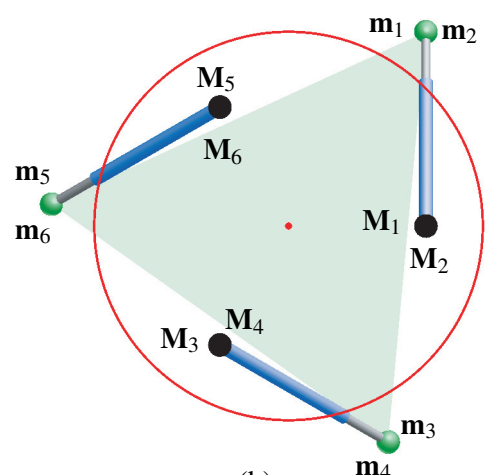

(b)

Fig. 1 Non-planar manipulator with cylindrical singularity surface: (a) Axonometric view. (b) Projection direction is $p$ : The singularity surface (with respect to the barycenter of the platform) is displayed as conic.

\section{Preliminary considerations}

The set of Stewart Gough Platforms whose singularity set for any orientation is a cylindrical surface with rulings parallel to a given direction $p$ also contains the set of architecture singular manipulators. This is due to the fact that the singularity surface of these manipulators equals the whole space of translations for any orientation.

It can easily be seen from the following example that the above two sets are distinct: The non-planar manipulator determined by $\mathbf{m}_{1}=\mathbf{m}_{2}, \mathbf{m}_{3}=\mathbf{m}_{4}, \mathbf{m}_{5}=\mathbf{m}_{6}$ and $\overline{\mathbf{M}_{1} \mathbf{M}_{2}} \|$ $\overline{\mathbf{M}_{3} \mathbf{M}_{4}}\left\|\overline{\mathbf{M}_{5} \mathbf{M}_{6}}\right\| p$ has for any orientation of the platform a cylindrical surface with rulings parallel to the direction $p$ without being architecturally singular (see Fig. 1). This manipulator is only in a singular configuration iff the three planes $\left[\mathbf{M}_{1}, \mathbf{M}_{2}, \mathbf{m}_{1}\right],\left[\mathbf{M}_{3}, \mathbf{M}_{4}, \mathbf{m}_{3}\right]$ and $\left[\mathbf{M}_{5}, \mathbf{M}_{6}, \mathbf{m}_{5}\right]$ have a common intersection line.

As the direct kinematics of this manipulator can be put down to that of a 3-dof RPR parallel manipulator, a rational parametrization of its singularity surface according to [1] can be given. The singularity surface is a quadratic cylinder due to the (singular) affine correspondence between the base and the platform (cf. [3]).

Moreover, if $\mathbf{M}_{1}, \ldots, \mathbf{M}_{6}$ are coplanar we get an example for a planar parallel manipulator with this property. Now the question arises, if there also exist non-architecturally singular planar manipulators with no four anchor points on a line possessing such a singularity surface. In the following section we prove that such manipulators do not exist.

\section{The main theorem and its proof}

Theorem The set of planar parallel manipulators with no four anchor points on a line which possess a cylindrical singularity surface with rulings parallel to a given fixed direction $p$ for any orientation of the platform equals the set of planar architecture singular manipulators (with no four anchor points on a line).

The analytical proof of this theorem is based on the following idea: We choose an Cartesian frame in the base such that one axis $t_{i}$ is parallel to the given direction $p$. Then $Q:=\operatorname{det}(\mathbf{Q})=0$ must be independent of $t_{i}$ for all $e_{0}, \ldots, e_{3}, t_{j}, t_{k}$ with $j \neq k \neq i \neq j$. Our proof is based on the resulting equations and Theorem 1 of Karger [2].

We have to distinguish between two cases given in the following subsections. 


\subsection{Base is not parallel to $p$}

The proof of the case where the base is orthogonal to $p$ is hidden in the proof of Theorem 1 given by Karger [2]. Therefore this case which corresponds to $C_{2}=C_{3}=0$ by eliminating $t_{3}$ from $Q$ needs not be discussed.

For all other directions we start analogously to Karger's proof by setting $t_{1}=t_{2}=0$ and performing the same elementary operations with the matrix $\mathbf{Q}$ as described on page 1154 of the cited paper. Then the last row of $\mathbf{Q}$ is of the form

$$
\begin{array}{r}
\left(r_{11} K_{1}+r_{12} A_{2} K_{2}, r_{21} K_{1}+r_{22} A_{2} K_{2}, r_{31} K_{1}+r_{32} A_{2} K_{2}, r_{21} C_{2} K_{3}+r_{22} C_{2} K_{4}\right. \\
\left.r_{31} A_{2} K_{3}+r_{32} A_{2} K_{4}-r_{11} C_{2} K_{3}-r_{12} C_{2} K_{4},-r_{21} A_{2} K_{3}-r_{22} A_{2} K_{4}\right) D^{-1}
\end{array}
$$

with $D:=A_{2} B_{3} B_{4} B_{5} \operatorname{coll}(3,4,5)$ and $r_{i j}$ of Equ. (1). It should be noted that $K_{1}=K_{2}=$ $K_{3}=K_{4}=0$ are the four conditions given in [2] which are satisfied iff a planar manipulator (with no four points on a line) is architecturally singular.

Now $Q$ can be written as

$$
Q=A_{2}^{2}\left(r_{11} r_{22}-r_{12} r_{21}\right) Q_{3} t_{3}^{3}+A_{2} B_{3} Q_{2} t_{3}^{2}+Q_{1} t_{3}+Q_{0} .
$$

With the coefficients $Q_{1}, Q_{2}$ and $Q_{3}$ the steps (a) and (b) on page 1155 of [2] can be done one by one. The steps $(\mathbf{c})$ and $(\mathbf{d})$ are different and therefore given here:

Step (c) $K_{1}=0, K_{2}=0, K_{4} \neq 0$

After substituting Euler parameters $e_{i}$ into $Q_{1}$ and $Q_{2}$, we can factor out $K$ of $Q_{i}(i=1,2)$; let us call the remaining coefficient again $Q_{i}(i=1,2)$.

(A) Let $B_{4} b_{3}-B_{3} b_{4} \neq 0$.

From the coefficient of $e_{0}^{2}$ in $Q_{2}$ we express $A_{5}$. Denote the coefficients of $e_{0}^{5} e_{1}$ and $e_{2}^{5} e_{3}$ of $Q_{2}$ by $v_{1}$ and $v_{2}$, respectively, and express $a_{5}$ from $v_{1}+v_{2}$. Now $B_{3}-b_{3}=0$ or $B_{4}-b_{4}=0$ must be different from zero; we may suppose $B_{3}-b_{3} \neq 0$. Therefore we can express $a_{4}$ from $v_{1}=0$. The coefficient $e_{0}^{1} e_{1}^{5}$ of $Q_{2}$ yields $a_{3}=a_{2} A_{3} / A_{2}$. Now the coefficients of $e_{0}^{4} e_{2} e_{3}$ and $e_{0} e_{1} e_{2}^{4}$ of $Q_{2}$ can only vanish (without contradiction) if $K_{3}=0$. The coefficient $e_{0}^{3} e_{3}^{3}$ of $Q_{2}$ yields $C_{3}=C_{2} A_{3} / A_{2}$. Finally we get as coefficient of $e_{0}^{3} e_{1}^{2} e_{2}$ of $Q_{2}$ the expression $A_{2} B_{3} K_{4} a_{2} \operatorname{coll}(3,4,5)$, a contradiction.

(B) Let $B_{4} b_{3}-B_{3} b_{4}=0$, i.e. $b_{4}=b_{3} B_{4} / B_{3}$.

From $v_{1}+v_{2}=0$ we get $b_{5}=b_{3} B_{5} / B_{3}$. Let us denote the coefficients of $e_{0} e_{1}^{5}, e_{1} e_{3}^{5}, e_{0}^{5} e_{2}$ of $Q_{2}$ by $v_{3}, v_{4}, v_{5}$. From $v_{2}-v_{3}=0$ and $v_{4}+v_{5}=0$ we compute $A_{4}$ and $A_{5}$. Now $Q_{1}$ factorizes into $K A_{2} F_{1}[16] F_{2}[2316] /\left(a_{2} B_{3}\right)$, where the number in the square brackets denotes the number of additive factors in the expression.

- ad $F_{1}=0$ : From the coefficient of $e_{0} e_{3}$ we express $a_{3}=a_{2} A_{3} / A_{2}$. If we denote the coefficients of $e_{i}^{2}$ by $q_{i}$, the sum $q_{0}+q_{1}+q_{2}+q_{3}$ yields $A_{2} B_{3}$, a contradiction.

- ad $F_{2}=0$ : We denote the coefficients of $e_{0}^{3} e_{1}, e_{0} e_{1}^{3}, e_{0}^{2} e_{1} e_{3}$ and $e_{1} e_{2}^{2} e_{3}$ by $p_{1}, p_{2}, p_{3}$ and $p_{4}$. The equations $p_{1}+p_{2}=0$ and $p_{3}-p_{4}=0$ can only vanish (without contradiction) if $K_{3}=0$ or $C_{2}=C_{3}=0$. As the later case can be neglected we set $K_{3}$ equal to zero. The equation $w_{1}-w_{2}=0$ vanishes (without contradiction) for $C_{2}=0$ or $F_{3}[12]=0$, where $w_{1}$ and $w_{2}$ are the coefficients of $e_{0}^{4}$ and $e_{1}^{4}$. If $C_{2}=0$ we obtain $C_{3}=0$ from $w_{1}+w_{2}=0$.

(i) Let $n:=B_{3} B_{4} a_{5}\left(a_{4}-a_{3}\right)+B_{3} B_{5} a_{4}\left(a_{3}-a_{5}\right)+B_{4} B_{5} a_{3}\left(a_{5}-a_{4}\right) \neq 0$. Then we can express $a_{2}=d / n$ from $F_{3}[12]=0$ with

$$
d:=a_{3}^{2} B_{4} B_{5}\left(a_{5}-a_{4}\right)+a_{4}^{2} B_{3} B_{5}\left(a_{3}-a_{5}\right)+a_{5}^{2} B_{3} B_{4}\left(a_{4}-a_{3}\right) .
$$


From the coefficient of $e_{0}^{3} e_{1}$ we compute $C_{2}$. Plugging the obtained expression into $F_{2}$ yields $K_{4} B_{4} B_{5} b_{3} F_{4}[80] F_{5}[96] /\left(A_{2} d n\right)$. Now the coefficient of $e_{0} e_{1}$ of $F_{4}[80]$ as well as the one of $F_{5}[96]$ yields $A_{2} B_{3} d=0$, a contradiction.

(ii) Let $n=0$. For $h:=B_{3} a_{4}\left(a_{5}-a_{3}\right)+B_{4} a_{3}\left(a_{4}-a_{5}\right) \neq 0$ we can compute $B_{5}$ from $n=0$. Substituting this into $F_{3}[12]$ yields the contradiction. If $h=0$ we can compute $a_{5}$ from this equation under the assumption $B_{3} a_{4}-B_{4} a_{3} \neq 0$. Substituting this into $n=0$ yields

$$
a_{4} a_{3} B_{4} B_{3}\left(a_{4}-a_{3}\right)\left(B_{3}-B_{4}\right) /\left(B_{3} a_{4}-B_{4} a_{3}\right)=0 .
$$

Now we have to distinguish between the following two cases:

$\star a_{3}=0$ or $a_{4}=0$ : Without loss of generality we assume $a_{3}=0$ and $a_{4} \neq 0$. Now the coefficient of $e_{1}^{2} e_{2} e_{3}$ of $F_{2}$ can only vanish (without contradiction) if $A_{2} B_{3} a_{4}-B_{3} a_{2} A_{2}+$ $B_{4} a_{2} A_{3}=0$ or $C_{2}=0$. For the later we obtain $C_{3}=0$ from $w_{1}=0$. Therefore we set $a_{4}=$ $a_{2}\left(B_{3} A_{2}-B_{4} A_{3}\right) /\left(A_{2} B_{3}\right)$ and substitute this into $w_{1}=0$. This equation can only vanish (without contradiction) for $C_{3}=0$. The coefficient of $e_{0}^{2} e_{1}^{2}$ of $F_{2}$ yields the contradiction.

$\star B_{3}-B_{4}=0$ : Substitution $B_{3}=B_{4}$ into $F_{3}[12]$ yields $B_{4} B_{5} a_{3} a_{4}\left(a_{3}-a_{4}\right)$ and therefore the above case; i.e. $a_{3}=0$ or $a_{4}=0$.

The last missing case is $B_{3} a_{4}-B_{4} a_{3}=0$. Plugging $a_{3}=a_{4} B_{3} / B_{4}$ into $h=0$ yields the contradiction. This finishes step $(c)$.

Step (d) $K_{1}=0, K_{2}=0, K_{4}=0, K_{3} \neq 0$

(A) Let $B_{3} b_{5}-b_{3} B_{5} \neq 0$.

We compute the coefficients $l_{i}$ of $e_{0}^{5} e_{2}, e_{0} e_{2}^{5}, e_{1}^{5} e_{3}$ and $e_{1} e_{3}^{5}$ of $Q_{2}$ which are of the form:

$$
\begin{array}{ll}
l_{1}=A_{2} B_{3} K_{3}\left(A_{2}-a_{2}\right) F_{6}[12], & l_{2}=A_{2} B_{3} K_{3}\left(A_{2}+a_{2}\right) F_{7}[12], \\
l_{3}=A_{2} B_{3} K_{3}\left(A_{2}-a_{2}\right) F_{7}[12], & l_{4}=A_{2} B_{3} K_{3}\left(A_{2}+a_{2}\right) F_{6}[12] .
\end{array}
$$

The equations $A_{2}-a_{2}=0$ and $A_{2}+a_{2}=0$ yield a contradiction.

(i) If we assume $b_{4} \neq 0$ we can compute $a_{4}$ and $A_{4}$ from $F_{6}[12]=0$ and $F_{7}[12]=0$. Now the sum of the coefficients of $e_{0}^{3} e_{3}^{3}$ and $e_{1}^{3} e_{2}^{3}$ of $Q_{2}$ yield $A_{2} C_{2} B_{3} K_{3} \operatorname{coll}(3,4,5)$ which implies $C_{2}=0$. The sum of the coefficients of $e_{0}^{4} e_{3}^{2}$ and $e_{1}^{2} e_{2}^{4}$ of $Q_{2}$ yield $A_{2} C_{3} a_{2} K_{3} \operatorname{coll}(3,4,5)$ and therefore $C_{3}=0$.

(ii) If $b_{4}=0$ we proceed similar and compute from $F_{6}=0$ and $F_{7}=0$ the unknowns $a_{4}$ and $A_{3}$. By performing the same steps as above we also obtain $C_{2}=C_{3}=0$.

(B) Let $B_{3} b_{5}-b_{3} B_{5}=0$, e.i. $b_{5}=b_{3} B_{5} / B_{3}$.

In this case we look at $l_{1}+l_{3}$ and $l_{1}+l_{4}$ which are of the form:

$$
l_{1}+l_{3}=C_{2} K_{3} b_{3} F_{8}[12] \text { and } l_{1}+l_{4}=b_{3}^{2} K_{3}\left(A_{3} C_{2}-A_{2} C_{3}\right) F_{9}[12] / B_{3}^{2},
$$

respectively. Moreover the linear combinations $m_{1}-m_{2}$ and $m_{1}+m_{3}$ are of the form

$$
m_{1}+m_{3}=C_{2} K_{3} b_{3}^{2} F_{9}[12] / B_{3} \quad \text { and } m_{1}-m_{2}=b_{3} K_{3}\left(A_{3} C_{2}-A_{2} C_{3}\right) F_{8}[12] / B_{3},
$$

where $m_{1}, m_{2}, m_{3}$ are the coefficients of $e_{0}^{2} e_{3}^{4}, e_{1}^{2} e_{2}^{4}$ and $e_{1}^{4} e_{2}^{2}$ of $Q_{2}$. As $C_{2}=C_{3}=0$ can be neglected we set $F_{8}[12]$ and $F_{9}[12]$ equal to zero and compute $A_{4}$ and $A_{5}$ from it. In the next step we factorize $Q_{1}$ which yields $A_{2} b_{3} B_{4} B_{5} K_{3} K F_{1}[16] F_{10}[144] \operatorname{coll}(3,4,5) /\left(a_{2} B_{3}\right)$.

(i) In step (c) it was already shown that $F_{1}[16]=0$ yields a contradiction.

(ii) Therefore we proceed by computing the sum of the coefficient of $e_{0}^{3} e_{1}$ and $e_{0} e_{1}^{3}$ of $F_{10}[144]$ which results in $a_{2} B_{3}^{2} C_{2}$. With $C_{2}=0$ the difference of the coefficient of $e_{0}^{3} e_{2}$ and $e_{0} e_{2}^{3}$ of $F_{10}[144]$ yield $a_{2} A_{2} B_{3}^{2} C_{3}$ and therefore $C_{3}=0$, which finishes this part. 


\subsection{Base is parallel to $p$}

In this case we take as translation vector $\mathbf{t}:=\left(\cos \varphi t_{1}-\sin \varphi t_{2}, \sin \varphi t_{1}+\cos \varphi t_{2}, t_{3}\right)^{T}$ and set $C_{2}=C_{3}=0$. After performing again the same elementary operations with the matrix $\mathbf{Q}$ as above and replacing the sixth row by Equ. (4), we have to distinguish between the following two cases.

\subsection{1 $\mathrm{M}_{1} \mathrm{M}_{2}$ is parallel to $p$}

If we set $\varphi=0$ the $t_{1}$ axis is parallel to $p$ ( $\Rightarrow Q$ must be independent of $t_{1}$ ). We denote the coefficients of $t_{1}^{i} t_{2}^{j} t_{3}^{k}$ from $Q$ by $Q^{i, j, k}$. From $Q^{1,0,1}$ we can factor out $K$ and from $Q^{1,0,0}$ we can even factor out $K^{2}$. We denote the coefficient of $e_{0}^{a} e_{1}^{b} e_{2}^{c} e_{3}^{d}$ of $Q^{i, j, k}$ by $P_{a, b, c, d}^{i, j, k}$ and compute

$$
\begin{aligned}
& P_{4,1,1,0}^{1,0,1}-P_{1,4,0,1}^{1,0,1}-P_{1,0,4,1}^{1,0,1}+P_{0,1,1,4}^{1,0,1}=K_{1} B_{3} B_{4} B_{5} \operatorname{coll}(3,4,5) \\
& P_{0,2,2,2}^{1,0,1}+P_{2,0,2,2}^{1,0,1}-P_{2,2,0,2}^{1,0,1}-P_{2,2,2,0}^{1,0,1}=K_{2} A_{2} B_{3} B_{4} B_{5} \operatorname{coll}(3,4,5)
\end{aligned}
$$

which yields $K_{1}=K_{2}=0$. Now we consider

$$
\begin{aligned}
& P_{3,1,2,0}^{1,0,0}-P_{2,0,3,1}^{1,0,0}-P_{1,3,0,2}^{1,0,0}+P_{0,2,1,3}^{1,0,0}=K_{3} a_{2} B_{3} B_{4} B_{5} \operatorname{coll}(3,4,5) \\
& P_{3,2,1,0}^{1,0,0}-P_{2,3,0,1}^{1,0,0}-P_{1,0,3,2}^{1,0,0}+P_{0,1,2,3}^{1,0,0}=K_{4} a_{2} B_{3} B_{4} B_{5} \operatorname{coll}(3,4,5)
\end{aligned}
$$

which finishes this part of the proof.

\subsection{2 $M_{1} M_{2}$ is not parallel to $p$}

As this part of the proof is too long to be presented here in its full length we refer to the corresponding technical report [7]. In the following we only give a sketch of the proof as well as the two special solutions $S_{1}$ and $S_{2}$ which cause difficulties.

First of all we can assume $\sin \varphi \neq 0$ if we eliminate $t_{1}$ from $Q$. If we assume additionally $K_{2}=0$, one can show in a similar way as above that also $K_{1}=K_{3}=K_{4}=0$ must hold. But if we assume $K_{2} \neq 0$ there exist two solutions which fulfill all equations resulting from the coefficients of $t_{1}$ of $Q$ without contradicting

$$
A_{2} B_{3} B_{4} B_{5} a_{2}\left(a_{4}-a_{3}\right) \operatorname{coll}(3,4,5) K_{2} \sin \varphi \neq 0
$$

These two solutions $S_{1}$ and $S_{2}$ are given by

$$
\begin{aligned}
S_{1}: \quad A_{i} & =B_{i} \cot \varphi, A_{j}=B_{j} \cot \varphi, A_{k}=A_{2}+B_{k} \cot \varphi, \\
b_{k} & =0, a_{2}=a_{k}, a_{i}=K_{1} b_{i} /\left(K_{2} A_{2}\right), a_{j}=K_{1} b_{j} /\left(K_{2} A_{2}\right), \\
K_{3} & =0 \text { and } K_{4}=0
\end{aligned}
$$

and

$$
\begin{array}{cl}
S_{2}: & A_{i}=A_{2}+B_{i} \cot \varphi, A_{j}=A_{2}+B_{j} \cot \varphi, A_{k}=B_{k} \cot \varphi \\
& a_{i}=a_{2}+b_{i} K_{3} / K_{4}, a_{j}=a_{2}+b_{j} K_{3} / K_{4}, a_{k}=b_{k}=0 \\
& A_{2} K_{2}+K_{4}=0 \text { and } K_{1}+K_{3}=0
\end{array}
$$

for $i, j, k \in\{3,4,5\}$ and $i \neq j \neq k \neq i$. In the following we show that these solutions also imply contradictions for the choice of $\mathbf{M}_{6}$ and $\mathbf{m}_{6}$, respectively. 
First of all we can set $A_{2}=1$ due to $A_{2} \neq 0$. Then we replace $K_{i}$ in Equ. (22) and (19) by the expressions given in Equ. (4) of [2]. If we plug now the expressions of Equ. (17) and (18) into the resulting equations of Equ. (19) we get

$$
K_{3}=\left(A_{6}-B_{6} \cot \varphi\right)\left(a_{k}-a_{6}\right) \quad \text { and } \quad K_{4}=\left(A_{6}-B_{6} \cot \varphi\right) b_{6},
$$

respectively. The solution $a_{6}=a_{k}$ and $b_{6}=0$ contradicts $K_{2} \neq 0$. If $A_{6}=B_{6} \cot \varphi$ the four base anchor points $\mathbf{M}_{1}, \mathbf{M}_{i}, \mathbf{M}_{j}$ and $\mathbf{M}_{6}$ are collinear.

For the second solution we proceed similarly, i.e. we plug the expressions of Equ. (20) and (21) into the equations of Equ. (22). We end up with

$$
K_{1}+K_{3}=\left(1-A_{6}+B_{6} \cot \varphi\right) a_{6} \quad \text { and } \quad K_{2}+K_{4}=\left(1-A_{6}+B_{6} \cot \varphi\right) b_{6} .
$$

The solution $a_{6}=b_{6}=0$ again contradicts $K_{2} \neq 0$. The other solution $A_{6}=1+B_{6} \cot \varphi$ implies the collinearity of the four base anchor points $\mathbf{M}_{2}, \mathbf{M}_{i}, \mathbf{M}_{j}$ and $\mathbf{M}_{6}$. This finishes the proof of the given Theorem.

\section{A further example}

The two solutions $S_{1}$ and $S_{2}$ imply a further example for an planar parallel manipulator with cylindrical singularity surface beside the one given in section 2 . The computation of the corresponding manipulator can be done as follows:

$S_{1}$ : If we set $A_{6}=B_{6} \cot \varphi$ there are two conditions left, which derive from Equ. (4) of [2].

Solving these two equations for the variables $K_{1}$ and $K_{2}$ yield:

$$
K_{1}=a_{6} s /\left(b_{6} B_{i} B_{j}\left(b_{i}-b_{j}\right)\right) \quad \text { and } \quad K_{2}=s /\left(B_{i} B_{j}\left(b_{i}-b_{j}\right)\right)
$$

with

$$
s:=B_{i} B_{j} b_{6}\left(b_{i}-b_{j}\right)+B_{j} B_{6} b_{i}\left(b_{j}-b_{6}\right)+B_{i} B_{6} b_{j}\left(b_{6}-b_{i}\right) .
$$

As special case we obtain

$$
\left.a_{6}=b_{6}=0 \quad \text { and } \quad K_{2}=b_{i} b_{j} B_{6}\left(B_{j}-B_{i}\right)\right) /\left(B_{i} B_{j}\left(b_{i}-b_{j}\right)\right) .
$$

$S_{2}$ : For $A_{6}=1+B_{6} \cot \varphi$ analogous computations yield

$$
K_{1}=s\left(a_{6}-a_{2}\right) /\left(b_{6} B_{i} B_{j}\left(b_{i}-b_{j}\right)\right), \quad K_{2}=s /\left(B_{i} B_{j}\left(b_{i}-b_{j}\right)\right),
$$

with $s$ of Equ. (26). Here the special case is given by

$$
a_{6}=a_{2}, b_{6}=0 \quad \text { and } \quad K_{2}=b_{i} b_{j} B_{6}\left(B_{j}-B_{i}\right) /\left(B_{i} B_{j}\left(b_{i}-b_{j}\right)\right) .
$$

It should be noted that we can assume $B_{i} B_{j}\left(b_{i}-b_{j}\right) \neq 0$, otherwise $D$ of Equ. (4) is equal to zero which is forbidden (division by zero).

Moreover it should be mentioned that if $s=0$ holds the manipulator is architecturally singular due to $K_{1}=K_{2}=K_{3}=K_{4}=0$. The condition $s=0$ expresses that the cross ratio of the base anchor points $\mathbf{M}_{x}, \mathbf{M}_{i}, \mathbf{M}_{j}, \mathbf{M}_{6}$ and of the corresponding platform anchor points $\mathbf{m}_{x}, \mathbf{m}_{i}, \mathbf{m}_{j}, \mathbf{m}_{6}$ is the same.

In the architecturally singular case the carrier lines of the involved four legs belong to a ruled quadric, which can also degenerate into two planes (cf. $8^{\text {th }}$ entry in the list of architecture singular Stewart Gough Platforms given by Karger in Theorem 3 of [4]). 


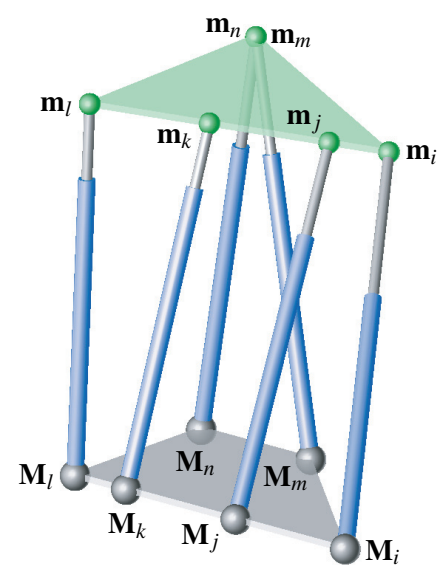

(a)

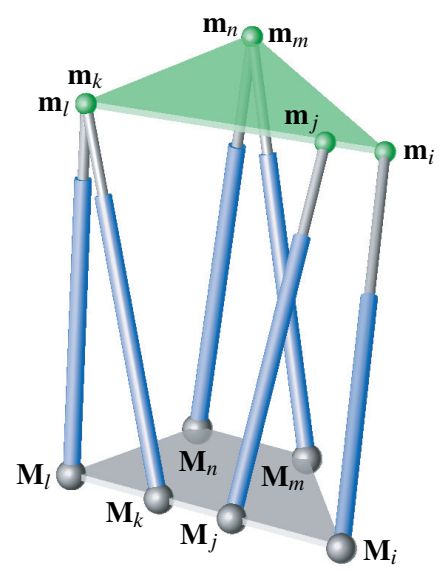

(b)

Fig. 2 Planar parallel manipulator with cylindrical singularity surface: (a) General case. (b) Special case.

It follows immediately from the expressions of $a_{i}$ and $a_{j}$ given in Equ. (18) and (21), respectively, that the platform anchor points $\mathbf{m}_{i}, \mathbf{m}_{j}$ and $\mathbf{m}_{x}$ of solution $S_{x}(x=1,2)$ are collinear. If we plug now the obtained expressions for $K_{1}$ and $K_{2}$ (given in Equ. (25) and (28), respectively) of solution $S_{x}$ into $a_{i}$ and $a_{j}$, we can see that also $\mathbf{m}_{6}$ is located on the line spanned by $\mathbf{m}_{i}, \mathbf{m}_{j}$ and $\mathbf{m}_{x}$. For both special cases (given in Equ. (27) and (29), respectively) this is trivially true due to $\mathbf{m}_{x}=\mathbf{m}_{6}$.

Therefore the geometric properties of the planar parallel manipulator with cylindrical singularity surface corresponding with solution $S_{1}$ and $S_{2}$ can be summarized as follows:
(i) $\mathbf{M}_{i}, \mathbf{M}_{j}, \mathbf{M}_{k}, \mathbf{M}_{l}$ are collinear,
(iii) $\overline{\mathbf{M}_{m} \mathbf{M}_{n}}\left\|\overline{\mathbf{M}_{i} \mathbf{M}_{j}}\right\| p$,
(ii) $\mathbf{m}_{i}, \mathbf{m}_{j}, \mathbf{m}_{k}, \mathbf{m}_{l}$ are collinear,
(iv) and $\mathbf{m}_{m}=\mathbf{m}_{n}$.

For the special cases we have the additional condition $\mathbf{m}_{k}=\mathbf{m}_{l}$. The manipulator and its special case is given in Fig. 2 (a) and (b), respectively.

This manipulator is in a singular position iff $\mathbf{m}_{m}=\mathbf{m}_{n}$ lies in the carrier plane of the base or if the carrier lines of $\mathbf{M}_{i}, \mathbf{M}_{j}, \mathbf{M}_{k}, \mathbf{M}_{l}$ and $\mathbf{m}_{i}, \mathbf{m}_{j}, \mathbf{m}_{k}, \mathbf{m}_{l}$ intersect each other. Therefore the quadratic singularity surface always splits into two planes (parallel to $p$ ).

\section{Remarks}

\section{Remark 1.}

The known examples of planar parallel manipulators with a cylindrical singularity surface (given in section 2 and section 4) raise the question if such manipulators with a cubic singularity surface exist. A complete list of planar parallel manipulators with a cylindrical singularity surface is in preparation [8].

\section{Remark 2.}

It should be noted that the proof of the second direction $\left(\operatorname{det}(\mathbf{Q})=0 \Rightarrow K_{1}=K_{2}=K_{3}=\right.$ $K_{4}=0$ ) of Theorem 1 given by Karger [2] can be replaced just by four equations, namely by Equ. (12-15). As the four conditions $K_{1}=K_{2}=K_{3}=K_{4}=0$ are expressed by not more than four equations, we have found the shortest possible analytical proof of the second direction of the cited theorem. 


\section{Remark 3.}

Röschel and Mick proved in [6,9] that planar parallel manipulators are architecturally singular iff $\left\{\mathbf{M}_{i}, \mathbf{m}_{i}\right\}$ for $(i=1, \ldots, 6)$ are four-fold conjugate pairs of points with respect to a 3 -dimensional linear manifold of correlations or one of the two sets $\left\{\mathbf{M}_{i}\right\}$ and $\left\{\mathbf{m}_{i}\right\}$ is situated on a line.

It would be nice to have such a geometric proof for the given theorem too. It might be possible to prove in a similar way to $[6,9]$ that planar parallel manipulators with no four points on a line and a cylindrical singularity surface must consist of four-fold conjugate pairs of anchor points.

\section{Conclusion}

We presented first results on planar parallel manipulators whose singularity set for any orientation is a cylindrical surface with rulings parallel to a given fixed direction $p$ in the space of translations. We proved that there do not exist non-architecturally singular Stewart Gough Platforms with planar base and platform and no four anchor points collinear which possess such a singularity surface.

As by-product of our proof we gave the shortest possible analytical proof for the second direction $\left(\operatorname{det}(\mathbf{Q})=0 \Rightarrow K_{1}=K_{2}=K_{3}=K_{4}=0\right)$ of Theorem 1 given by Karger [2] Moreover, we presented two examples of planar manipulators with cylindrical singularity surface. A complete list of such planar parallel manipulators is in preparation [8].

\section{References}

[1] Husty, M.L., Hayes, M.J.D., and Loibnegger, H.: The General Singularity Surface of Planar ThreeLegged Platforms, Advances in Multibody Systems and Mechantronics (A. Kecskemethy eds.), Duisburg, Germany, pp. 203-214 (1999).

[2] Karger, A.: Architecture singular planar parallel manipulators, Mechanism and Machine Theory 38 (11) 1149-1164 (2003).

[3] Karger, A.: Stewart-Gough platforms with simple singularity surface, Advances in Robot Kinematics: Mechanisms and Motion (J. Lenarcic, B. Roth eds.), 247-254, Springer (2006).

[4] Karger, A.: Architecturally singular non-planar parallel manipulators, Mechanism and Machine Theory 43 (3) 335-346 (2008).

[5] Merlet, J.-P.: Singular Configurations of Parallel Manipulators and Grassmann Geometry, International Journal of Robotics Research 8 (5) 45-56 (1992).

[6] Mick, S., and Röschel, O.: Geometry \& architecturally shaky platforms, Advances in Robot Kinematics: Analysis and Control (J. Lenarcic, M.L. Husty eds.), 455-464, Kluwer (1998).

[7] Nawratil, G.: Main Theorem on Planar Parallel Manipulators with Cylindrical Singularity Surface, Technical Report No. 187, Geometry Preprint Series, Vienna University of Technology (2008).

[8] Nawratil, G.: All Planar Parallel Manipulators with Cylindrical Singularity Surface, in preparation.

[9] Röschel, O., and Mick, S.: Characterisation of architecturally shaky platforms, Advances in Robot Kinematics: Analysis and Control (J. Lenarcic, M.L. Husty eds.), 465-474, Kluwer (1998). 